\title{
The relationship between the degree of brain edema regression and changes in cognitive function in patients with recurrent glioma treated with bevacizumab and temozolomide
}

\author{
Xianglian Wang ${ }^{1 \wedge}$, Di Chen ${ }^{1}$, Jianjian Qiu ${ }^{1}$, Shihong Li ${ }^{2}$ Xiangpeng Zheng ${ }^{1}$ \\ ${ }^{1}$ Department of Radiation Oncology, Fudan University Huadong Hospital, Shanghai, China; ${ }^{2}$ Imaging Center, Fudan University Huadong Hospital, \\ Shanghai, China
}

Contributions: (I) Conception and design: X Wang, X Zheng; (II) Administrative support: X Zheng; (III) Provision of study materials or patients: X Wang, S Li; (IV) Collection and assembly of data: X Wang, D Chen, S Li; (V) Data analysis and interpretation: D Chen, X Wang, J Qiu; (VI) Manuscript writing: All authors; (VII) Final approval of manuscript: All authors.

Correspondence to: Xianglian Wang; Xiangpeng Zheng, MD. Department of Radiation Oncology, Fudan University Huadong Hospital, 221 West Yan’an Road, Shanghai, China. Email: wangxianglian@fudan.edu.cn; Zhengxp@fudan.edu.cn.

Background: This retrospective study aims to assess the impacts on cognitive status and quality of life in recurrent high-grade glioma patients treated with temozolomide (TMZ), either alone or in combination with bevacizumab (BEV), and explore the relationship between the brain edema regression, BEV use, and cognitive status.

Methods: A total of 125 patients with recurrent high-grade glioma were enrolled in this study, of which 65 patients were treated with BEV (5-10 mg/ $\mathrm{kg}$ IV every 2 weeks) plus TMZ (200 mg/m² every 28 days, d1-5), and 60 patients were treated with TMZ $\left(200 \mathrm{mg} / \mathrm{m}^{2}\right.$ every 28 days, d1-5) alone. The treatment response was evaluated using the Response Assessment in Neuro-Oncology (RANO) criteria. Tumor-associated edema was evaluated with T2WI magnetic resonance imaging (MRI) and quantitative T2 mapping sequence, and an Edema Regression Index was designed to quantify volumetric changes in edema imaging after every treatment cycle. Cognitive intelligence state and quality of life were evaluated using the Mini-Mental State Examination (MMSE) and the European Organization for Research and Treatment of Cancer Quality of Life Questionnaire Core 30 (QLQ-C30).

Results: Radiologically, the partial response rate was $78.5 \%$ in the $\mathrm{BEV}+\mathrm{TMZ}$ group and $38.3 \%$ in the TMZ group. After the first cycle of treatment, the mean score of the MMSE was $21.1 \pm 2.0$ and $24.1 \pm 1.4$ $(\mathrm{P}<0.001)$ in the TMZ group and the BEV + TMZ group, respectively. In the functioning domains of the QLQ-C30, scales of physical functioning, emotional functioning and cognitive functioning were 43.0 \pm 7.0 vs. $61.7 \pm 12.5(\mathrm{P}<0.001), 44.5 \pm 8.8$ vs. $63.4 \pm 6.9(\mathrm{P}<0.001)$ and $42.4 \pm 8.8$ vs. $63.7 \pm 12.0(\mathrm{P}<0.001)$ in the TMZ group and the $\mathrm{BEV}+\mathrm{TMZ}$ group, respectively. In the BEV + TMZ group, a correlation between the Edema Regression Index and improvement in cognitive status and quality of life was observed. Patients with Edema Regression Index scores higher than 50\% gained a 25.6\% increase in the mean MMSE score from $19.9 \pm 1.6$ to $25.0 \pm 1.1(\mathrm{P}<0.001)$. In the $\mathrm{BEV}+\mathrm{TMZ}$ group, physical functioning, emotional functioning, and cognitive functioning increased by $76.8 \%, 53.1 \%$, and $81.5 \%$, respectively, while scores of nausea/vomiting decreased by $40.3 \%$ to 32.1 . Patients with no evident edema observed in the pre-BEV MRI scans were given a prolonged four-cycle course of BEV. No significant improvement was observed in the MMSE score and the QLQ score with additional cycles of BEV.

\footnotetext{
$\wedge$ ORCID: 0000-0002-3359-4293.
} 
Conclusions: A close relationship was observed between Edema Regression Index and a change in cognitive function in patients treated with BEV and TMZ. Compared with TMZ alone, the combination of TMZ and BEV could improve the cognitive function and quality of life of patients with recurrent high-grade gliomas. The Edema Regression Index could be used as a surrogate imaging biomarker to predict patients who may or may not gain cognitive benefit from the combination therapy of TMZ and BEV, which warrants further prospective clinical studies for validation.

Keywords: Cognition; brain edema; bevacizumab; glioma; quality of life

Submitted Sep 20, 2020. Accepted for publication Jun 22, 2021.

doi: 10.21037/qims-20-1084

View this article at: https://dx.doi.org/10.21037/qims-20-1084

\section{Introduction}

Gliomas, the most common primary brain tumor, originate from glial stem cells or progenitor cells and account for approximately $30 \%$ of all central nervous system tumors (1). The standard therapeutic regimen for newly diagnosed high-grade glioma (HGG) patients is maximal safe surgical resection followed by external beam radiotherapy with concurrent and adjuvant temozolomide (TMZ). Unfortunately, compared with low-grade gliomas, the median survival of glioblastoma patients is only 14 months, the 3 -year survival rate is approximately $15 \%$, and the 5 -year survival rate is less than $10 \%$ (2). Almost all HGG patients relapse after first-line treatment and have an extremely poor prognosis. There is currently no standard regimen for recurrent glioma, and there is typically only a few months between relapse and death. The development of tumors and brain edema worsens patients' quality of life, resulting in various neurological symptoms and cognitive dysfunction. Therefore, slowing the development of clinical symptoms and improving quality of life is particularly important for patients with recurrent glioma. Bevacizumab (BEV) was approved to treat recurrent HGG by the United States Food and Drug Administration in 2009 due to its benefits in improving tumor progression-free survival (3). However, there are still many concerns about BEV use, including the potential risk of tumor bleeding and ischemic stroke (4), the induction of tumor vascular mimicry, and effects on cognitive function. Therefore, the use of $\mathrm{BEV}$ needs to be further optimized and individualized, including the dosage, timing, and duration (or cycles). This study retrospectively analyzes the influence of $\mathrm{BEV}$ on cognitive function and neuroimaging changes in patients with recurrent HGG to explore the characteristics of patients who may or may not benefit from $\mathrm{BEV}$ to improve, or at least not compromise, the quality of life of these patients with limited survival time.

\section{Methods}

\section{Patients}

This study analyzes 125 patients who presented to Huadong Hospital with recurrent HGG between October 2014 and January 2020. The criteria for inclusion were as follows: (I) glioma was diagnosed based on histological examination and classified as grade III or IV; (II) the initial treatment included surgery, radiotherapy, and chemotherapy, but without bevacizumab; (III) glioma recurrence was confirmed by pathology or comprehensive imaging assessment to exclude pseudoprogression; (IV) recurrent lesions were measurable in magnetic resonance imaging (MRI); and (V) no bleeding inclination, with normal liver and kidney function. The exclusion criteria included: (I) newly diagnosed glioma without standard treatment; and (II) recent history of active hemorrhage or stroke. Antiepileptic drugs (sodium valproate) had been stopped for more than six months, and no patient had taken psychotropic drugs. Patient characteristics are shown in Table 1.

\section{Treatments}

As the salvage therapy for recurrent gliomas, 65 patients received the combination regimen of $\mathrm{BEV}$ (Avastin, Roche, Switzerland) plus TMZ (Temodal, Merck Sharp \& Dohme Ltd, UK), and 60 patients received TMZ alone due to certain contraindications to $\mathrm{BEV}$ (such as gastric ulcer, poor control of hypertension, thrombosis, etc.). In the TMZ group, oral TMZ $\left(200 \mathrm{mg} / \mathrm{m}^{2}\right)$ was given on day 1 through 5 every 28 days, while the combination group was given the TMZ treatment plus BEV $(5-10 \mathrm{mg} / \mathrm{kg})$ 
Table 1 Patient demographics and baseline characteristics

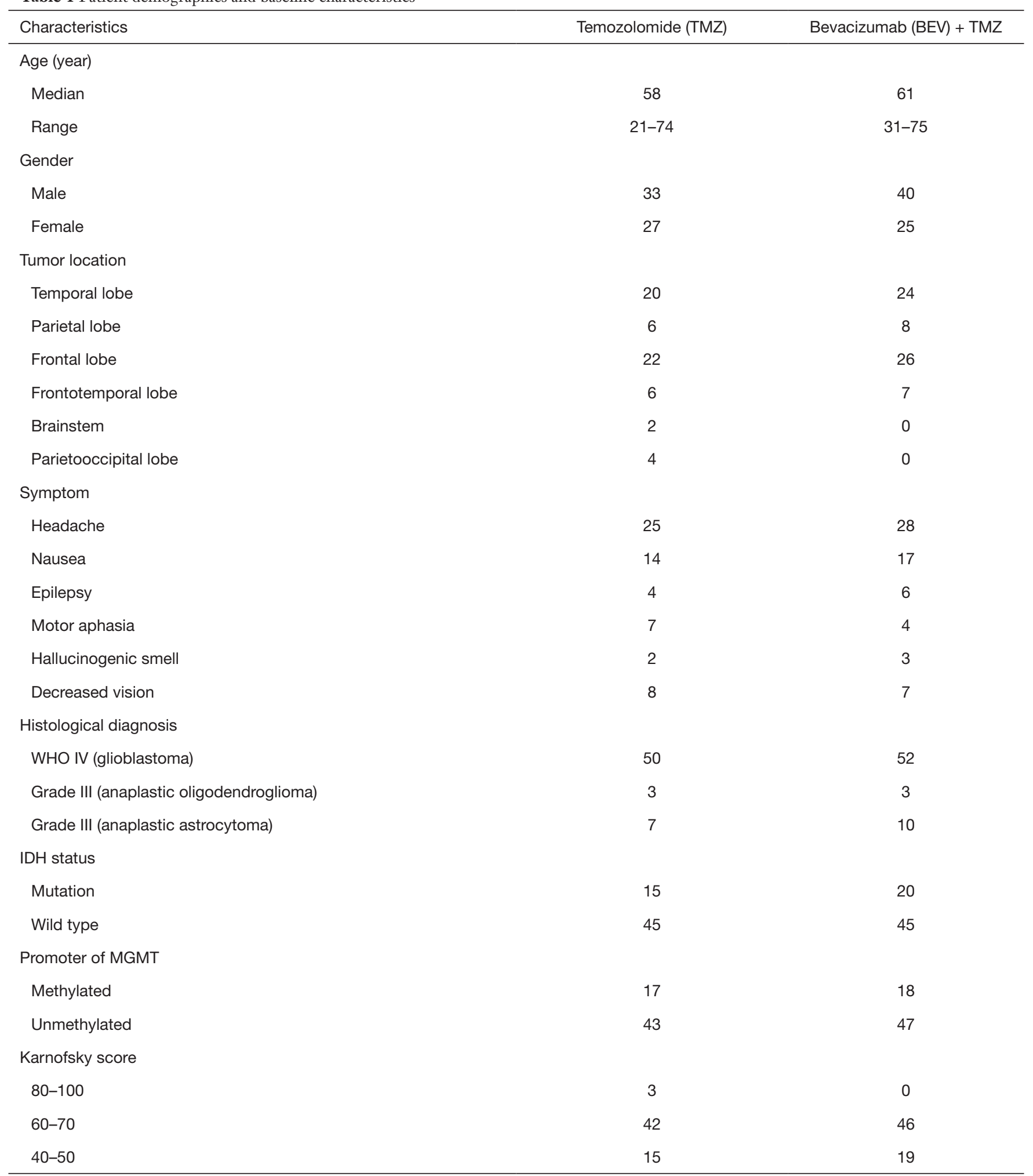

Table 1 (continued) 
Table 1 (continued)

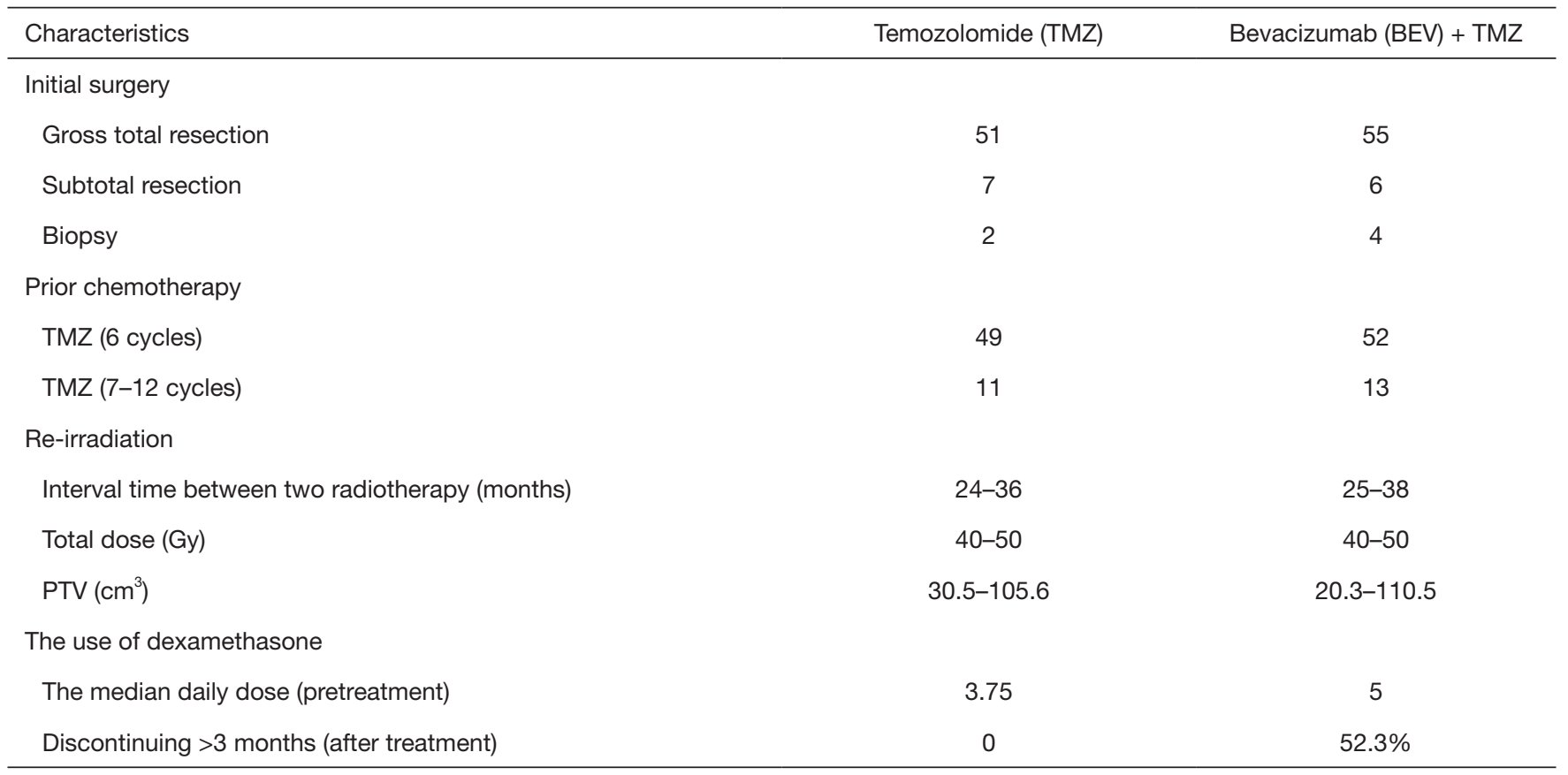
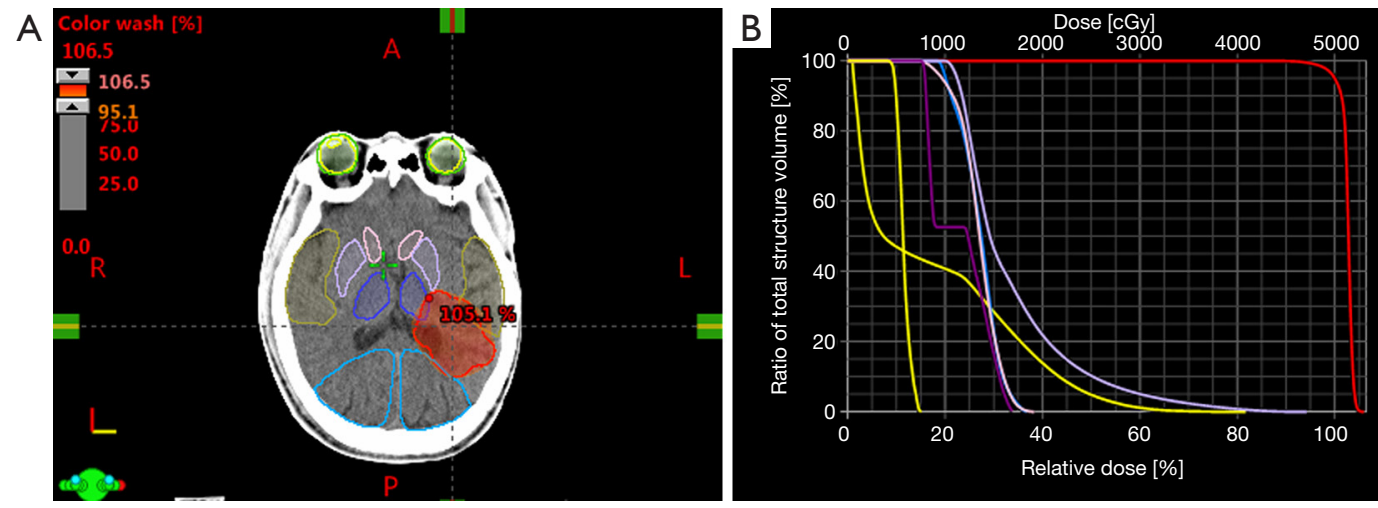

Figure 1 Treatment planning system, showing the contours and dose of planning target volume (PTV) and organ at risk (OAR).

administered intravenously every 2 weeks. Each four-week treatment cycle included 5-day TMZ with or without 2 doses of BEV. During the first radiotherapy, 60 Gy was delivered in 30 fractions. Eighteen patients received reirradiation. All patients were treated with the intensitymodulated radiotherapy technique (Figure 1); computed tomography simulation was performed in a supine position with thermoplastic mask immobilization. $6 \mathrm{MV} \mathrm{X}$-rays were used. The gross tumor volume (GTV) was defined as the abnormal signal region on enhanced T1-weighted imaging, and the clinical target volume (CTV) was contoured as the
GTV plus $0.3 \mathrm{~cm}$. The planning target volume (PTV) was contoured as the CTV plus $0.2 \mathrm{~cm}$ and prescribed 40-50 Gy in 15-25 fractions. All patients underwent MRI scans before treatment and two weeks after the first, second, and third cycle. Routine blood tests, liver and kidney function, coagulation factors, and blood pressure were checked before each treatment cycle.

\section{Cognitive function evaluation}

The Mini-Mental State Examination (MMSE) is a 
commonly used questionnaire for assessing cognitive impairment, typically taking 5-10 minutes to complete (5). However, when interpreting the test results, examiners or physicians should consider patient demographic factors (especially age and education). The MMSE quantitatively measures orientation, short-term memory (but not longterm memory), attention, and language functioning. Scores of 25-30 out of 30 are considered normal; the National Institute for Health and Care Excellence (NICE) classifies 21-24 as mild scores, scores of 10-20 as moderate, and scores $<10$ as a severe impairment. Before administering the MMSE, it is important to make the patient comfortable and to establish rapport. This assessment was performed before treatment and two weeks after each cycle of BEV. The Montreal cognitive assessment (MoCA) (6) was used to evaluate the validity of MMSE. A neuropsychologist and a neurologist performed the questionnaires of MMSE and MoCA. Routine assessments continued for two or more years after all treatments.

\section{Treatment response}

Treatment response was assessed using the Response Assessment in Neuro-Oncology (RANO) criteria based on changes in the T1-weighted gadolinium-enhanced imaging and non-enhanced T2/FLAIR sequence (7). Complete response (CR) was defined as the disappearance of tumor signal; partial response (PR) was defined as a $\geq 50 \%$ decrease in the bidirectional tumor area on contrast enhancement; stable disease (SD) was defined as a $<50 \%$ decrease or a $<25 \%$ increase in tumor area; progressive disease (PD) was defined as a $\geq 25 \%$ increase in the tumor area. Overall response (OR) included CR and PR. PFS and OS were calculated from the start of the combined treatment. In assessing the effect of the combination therapy on brain edema, the Edema Regression Index (ERI) was introduced to quantitate the change in edema volume before and after the BEV treatment. The image series from the MRI T2FLAIR and T1 enhanced images were input into the radiation oncologist workstation with the treatment planning system (TPS). The edema boundary $\left(\mathrm{PTV}_{\mathrm{E}}\right.$, including the tumor and edema T2FLAIR/T2WI/ T2 mapping) and the GTV (T1 enhanced image) boundary were delineated slice-by-slice using the contouring tool, and the volume for each structure was derived. The actual edema volume was calculated as follows: $\left(\mathrm{V}_{\text {edema }}\right)=\mathrm{PTV}_{\mathrm{E}^{-}}$ $\mathrm{GTV}$, and ERI $=1-\left[\mathrm{V}_{\text {edema }}(\right.$ after $\mathrm{BEV}) / \mathrm{V}_{\text {edema }}$ (before $\mathrm{BEV})]$.

\section{Magnetic resonance imaging}

Data were collected on a 3-T MR system (MAGNETOM Prisma, Siemens Healthineers, Erlangen, Germany) using a 20-channel head and neck coil. MRI sequences included axial T2 weighted turbo spin-echo (TE/TR $=92 \mathrm{~ms} / 4,000 \mathrm{~ms}$, slice thickness $=5 \mathrm{~mm}$ with $30 \%$ inter-slice distance, averages $=1$, matrix size $=384 \times 384$, and FOV $=230 \mathrm{~mm}$ ). Quantitative T2 maps were generated from a standard spin echo sequence with ten echoes $(\mathrm{TE} / \mathrm{TR}=10.5,21,31.5,42,52.5,63$, $73.5,84,94.5,105 \mathrm{~ms} / 2,000 \mathrm{~ms}$, slice thickness $=4 \mathrm{~mm}$ with $20 \%$ inter-slice distance, averages $=1$, matrix size $=256 \times 256$, and $\mathrm{FOV}=250 \mathrm{~mm})$. Region of interest $(\mathrm{ROI})$ was selected in the area of long T2 on T2 weighted imaging (T2WI) sequence, which overlapped with differential quantitative T2 mapping. T2 was measured within a $5 \mathrm{~mm}$ diameter spherical ROI pretreatment and two weeks after the second and the fourth cycle of BEV to verify the validity of the ERI. Perfusion-weighted imaging (PWI), diffusionweighted imaging (DWI), magnetic resonance spectroscopy (MRS), and contrast-enhanced T1-weighted imaging (CET1WI) were used to differentiate true progression from pseudoprogression.

\section{Quality of life assessment}

The European Organization for Research and Treatment of Cancer (EORTC) Quality of Life Questionnaire Core 30 (QLQ-C30) (version 3.0) was applied to assess the healthrelated quality of life (HRQL) (8). The QLQ-C30, the core HRQL questionnaire, is frequently used to assess a range of functional outcomes and symptoms relevant to a wide range of cancer populations. The QLQ-C30 is a 30-item, self-reported questionnaire consisting of fifteen domains as follows: physical functioning (five items), role functioning (two items), emotional functioning (four items), cognitive functioning (two items), social functioning (two items), global health status (two items), fatigue (three items), pain (two items), nausea/vomiting (two items) and single items for dyspnea, insomnia, anorexia, constipation, diarrhea, and financial impact. The patients received the assessment before treatments and two weeks after each cycle of treatment.

\section{Statistics}

Survival analysis was performed using the Kaplan-Meier method. Scores of the MMSE, MoCA, and QLQ-C30 
were expressed as the mean \pm standard deviation (SD). The difference between the MMSE score and the mean score of each item of QLQ-C30 before treatment and after the second cycle of BEV was compared with the paired t-test. Linear regression was used to assess the relationship between the change of MMSE and ERI. Statistical analysis was performed using SPSS software (version 19.0), and the $P$ value less than 0.05 was considered statistically significant.

The study was conducted following the Declaration of Helsinki (as revised in 2013). The Ethics Committee approved the study of Clinical Research of Huadong Hospital. Written informed consent from the patients/ participants was not required to participate in this study in accordance with the national legislation and the institutional requirements.

\section{Results}

\section{Patient characteristics and response to the treatment}

A total of 125 patients met the inclusion criteria and were enrolled in this study, comprising 73 males and 52 females with a median age of 60 years (21-75 years). The average Karnofsky Performance Score (KPS) before treatment was 60. Anatomically, 48 tumors were located in the frontal lobe, 44 in the temporal lobe, 14 in the parietal lobe, 13 in the frontotemporal lobe, 2 in the brainstem, 4 in the parietooccipital lobe. The patients experienced various symptoms, such as headache, nausea, hallucinogenic smell, and decreased vision with the development of recurrent lesions. In all, $52.3 \%$ of the patients in the BEV + TMZ group and $0 \%$ in the TMZ group were able to discontinue corticosteroids for more than 3 months (Table 1).

Regarding the response rate, CR and PR were achieved in $3.1 \%$ and $78.5 \%$ of the patients in the BEV + TMZ group, respectively (Figure 2), resulting in an OR of $81.6 \%$. SD was observed in the rest of the patients and no progression occurred by the end of treatment. The median PFS and OS were 9 months and 12 months, respectively. The 12 -month PFS and OS were $25.6 \%$ and $51.7 \%$, respectively. In the TMZ group, PR was seen in $38.3 \%$ of the patients, SD in $20 \%$, and PD in $41.7 \%$.

\section{Change in cognitive function and the relationship with ERI}

All patients comprehended the contents of the MMSE and completed the assessment in 10 minutes. After the first cycle of treatment, the mean score of the MMSE was $21.1 \pm 2.0$ and $24.1 \pm 1.4(\mathrm{P}<0.001)$ in the TMZ group and the BEV + TMZ group, respectively. In the BEV + TMZ group, the mean $V_{\text {edema }}$ decreased from 180.0 to $50.0 \mathrm{~cm}^{3}$, the mean score of the MMSE increased from $20.3 \pm 1.4$ to $24.1 \pm 1.4$ $(\mathrm{P}<0.001)$, and the mean score of the MoCA increased from $20.2 \pm 1.3$ to $23.7 \pm 1.9(\mathrm{P}<0.001)$.

After the first cycle of treatment, the mean ERI was 0.23 (range: $0.05-0.39$ ) in the TMZ group, and no patient achieved significant edema remission (defined by ERI $>0.5$ ). The mean ERI was 0.56 (range: $0.20-0.81$ ) in the $\mathrm{BEV}+\mathrm{TMZ}$ group, and the improvement in the MMSE was closely correlated with the ERI. Patients with ERI higher than 0.5 had a $25.6 \%$ increase from $19.9 \pm 1.6$ to $25.0 \pm 1.1$ in the mean MMSE score $(\mathrm{P}<0.001)$ (Figure 3). The mean score of the MMSE in Patients with ERI lower than 0.5 changed from $21.1 \pm 1.4$ to $22.2 \pm 2.1(\mathrm{P}=0.06)$. The relationship between the change of MMSE and ERI was evaluated by linear regression $(\mathrm{R}=0.96, \mathrm{P}<0.001)$.

The region of brain edema in T2WI was consistent with that in the DQT2 mapping sequence (Figure 4). After two cycles of BEV, median $\mathrm{T} 2$ within pretreatment $\mathrm{T} 2$ signal abnormality was significantly reduced from $164.8 \pm 27.9$ to $100.5 \pm 6.2 \mathrm{~ms}(\mathrm{P}<0.001)$ in patients with ERI higher than 0.5 in the BEV + TMZ group.

However, in 37 patients who received four or more cycles of BEV, the improvement in cognitive function did not continue after the fourth cycle of $\mathrm{BEV}$ with comparison to the cognitive scores after the second cycle of $\mathrm{BEV}$, with a mean score of $24.1 \pm 1.1$ vs. $23.8 \pm 1.8(\mathrm{P}=0.31)$. The symptoms in 8 patients slightly deteriorated than those at the end of the second cycle of BEV. The $V_{\text {edema }}$ remained stable between the second and fourth cycle of BEV.

\section{Changes in quality of life and the relationship with ERI}

Patients with recurrent HGG not only carried a high symptom burden, such as headache, nausea, blurred vision, emotional changes, and speech and cognition disorders but also had significant difficulties in their performance of several domains of life. The QLQ-C30 was chosen to assess the function, symptoms, and quality of life.

In the functioning domains of the QLQ-C30, physical functioning $(\mathrm{PF})$, emotional functioning $(\mathrm{EF})$ and cognitive functioning (CF) after the first cycle of treatment were $43.0 \pm 7.0$ vs. $61.7 \pm 12.5(\mathrm{P}<0.001), 44.5 \pm 8.8$ vs. $63.4 \pm 6.9$ $(\mathrm{P}<0.001)$ and $42.4 \pm 8.8$ vs. $63.7 \pm 12.0(\mathrm{P}<0.001)$ in the $\mathrm{TMZ}$ group and the $\mathrm{BEV}+\mathrm{TMZ}$ group, respectively (Figure 5). In the BEV + TMZ group, the scores of PF, 

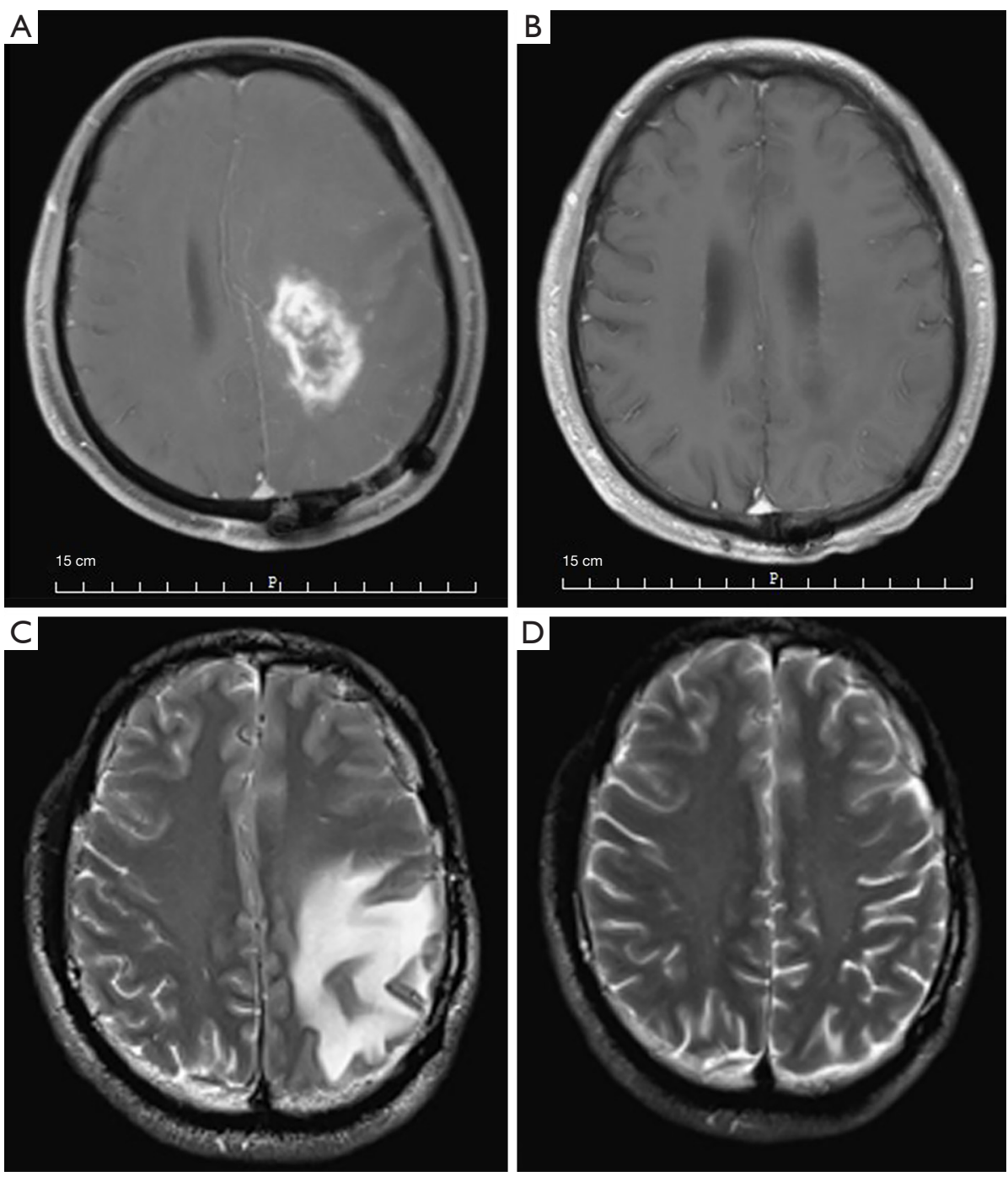

Figure 2 Changes of tumor and edema after the second cycle of bevacizumab (BEV) compared to base line in the BEV + temozolomide (TMZ) group. (A) The tumor pretreatment in the contrast T1-weighted image; (B) The tumor enhancement disappeared after the second cycle of BEV in the contrast T1-weighted image; (C) The cerebral edema distribution pretreatment in the T2-weighted image; (D) The cerebral edema alleviated visibly after the second cycle of BEV in the T2-weighted image.

EF and CF significantly improved after the first cycle of treatment compared to the baseline values, with the mean scores of $61.7 \pm 12.5$ vs. $34.9 \pm 12.0(\mathrm{P}<0.001), 63.4 \pm 6.9$ vs. $41.4 \pm 9.2(\mathrm{P}<0.001)$, and $63.7 \pm 12.0$ vs. $35.1 \pm 13.1(\mathrm{P}<0.001)$, respectively. In the symptom domains, the scores of pain and nausea/vomiting decreased significantly compared to those seen at baseline evaluation, from $46.4 \pm 23.3$ to $26.8 \pm 10.5(\mathrm{P}<0.001)$ and $53.8 \pm 15.1$ to $32.1 \pm 11.5(\mathrm{P}<0.001)$, respectively. The global health status also improved from $39.8 \pm 6.9$ to $69.0 \pm 12.2(\mathrm{P}<0.001)$ (Table 2).

In patients with ERI higher than 0.5 in the BEV + TMZ group, $\mathrm{PF}$ and $\mathrm{EF}$ changed from $30.6 \pm 7.0$ and $39.1 \pm 7.3$ to $64.5 \pm 8.6$ and $66.1 \pm 4.8$, respectively $(\mathrm{P}<0.001)$. In patients with ERI less than $0.5, \mathrm{PF}$ and $\mathrm{EF}$ changed from $38.5 \pm 11.3$ and $44.4 \pm 10.9$ to $45.3 \pm 8.2(\mathrm{P}=0.07)$ and $50.3 \pm 8.5(\mathrm{P}=0.06)$, respectively.

In agreement with the MMSE-based cognitive assessment, the QLQ-C30 based physical and cognitive functioning improvement did not extend after the fourth cycle of BEV with comparison to the values obtained after the second cycle, with mean scores of $66.3 \pm 13.2 \mathrm{vs}$. $65.9 \pm 12.4(\mathrm{P}=0.83)$ and $64.6 \pm 11.6$ vs. $63.6 \pm 12.5(\mathrm{P}=0.68)$, 


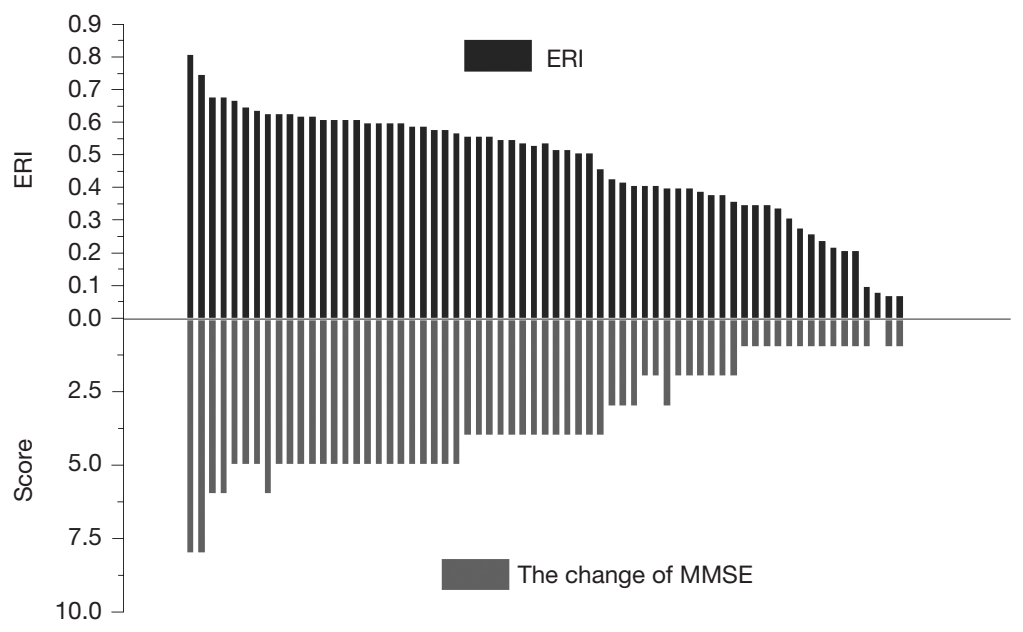

Figure 3 The relationship between the change of the Mini-Mental State Examination (MMSE) and edema regression index (ERI). MMSE score increased significantly in the patients who have Higher ERI after one cycle of treatment in the BEV + TMZ group.
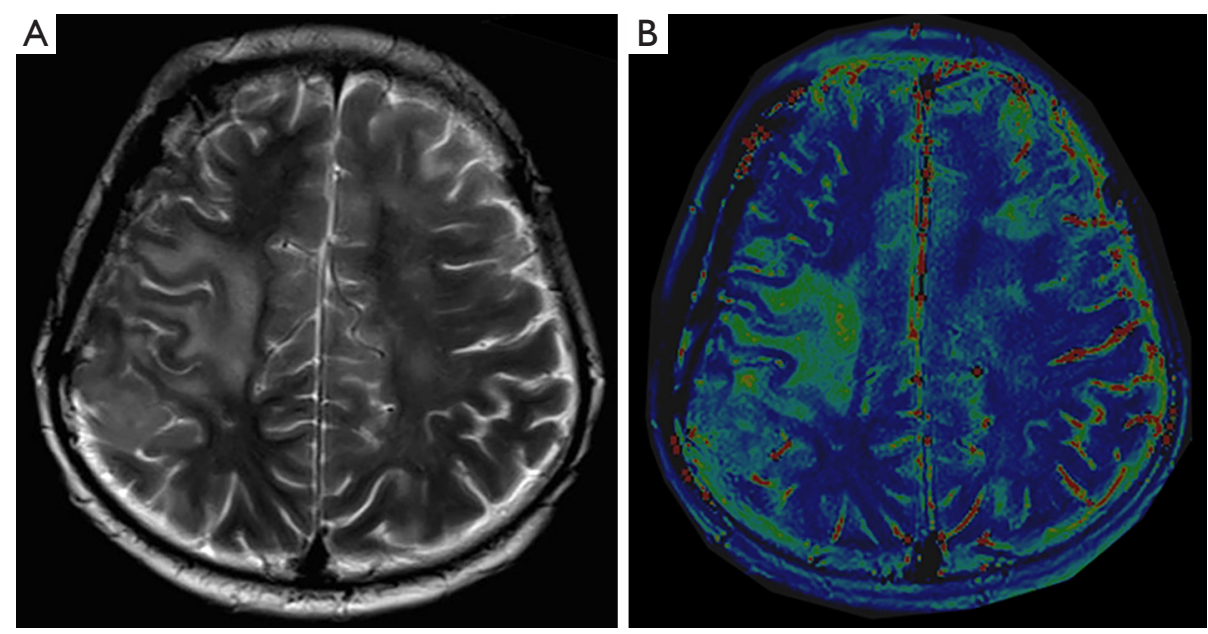

Figure 4 The abnormal signal region in T2 weighted imaging (T2WI) and differential quantitative T2 (DQT2) mapping. (A) High signal on T2WI; (B) the image on DQT2.

respectively.

\section{Adverse events}

During the treatment and follow-up, blood pressure, coagulation function, cranial MRI imaging, and liver and kidney functions were closely monitored. In the BEV + TMZ group, treatment-related hypertension was identified in 10 patients, liver dysfunction was observed in 8 patients, and thrombocytopenia was identified in 7 patients. In the TMZ group, liver dysfunction was observed in 7 patients, and thrombocytopenia was identified in 8 patients. These adverse effects were medically managed and did not interrupt the subsequent treatment cycle. Other reactions reported previously (9), such as intracranial hemorrhage, venous embolism events, poor wound healing, and gastrointestinal reactions were not observed in this study.

\section{Discussion}

Advances in molecular targeted therapy have provided enormous opportunities for long-term control of various 
malignancies, including recurrent gliomas. However, patients with recurrent HGGs are faced with a grave prognosis, characterized by a rapid decline in quality of life due to tumor aggressiveness and the ensuing intracranial pressure mainly from tumor-related edema. Therefore, besides the anti-tumor treatment, anti-edema therapy

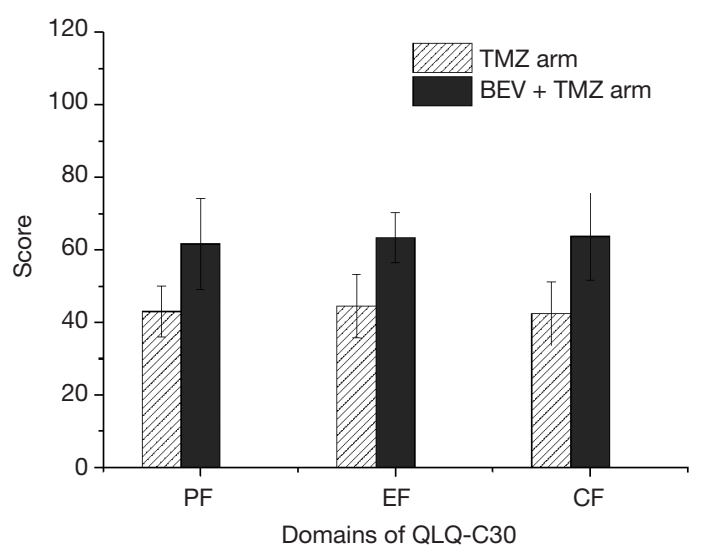

Figure 5 The comparison of scores of domains of QLQ-C30 between the $\mathrm{BEV}+\mathrm{TMZ}$ group and the TMZ group in patients with recurrent glioma after one cycle of TMZ. After one cycle of treatment, the score of PF, EF and CF increased more in the $\mathrm{BEV}+\mathrm{TMZ}$ group compared with the TMZ group. PF, physical function; CF, cognitive function; EF, emotional function; QLQ-C30, Quality of Life Questionnaire Core 30; BEV, bevacizumab; TMZ, temozolomide. appears extremely crucial in this patient population. As a neovasculature-rich malignancy, glioblastoma theoretically appears to be a good candidate tumor for anti-angiogenic therapy, represented by bevacizumab, a recombinant humanized IgGl monoclonal antibody. However, clinical trials showed negative results with the addition of BEV to standard therapy $(10,11)$.

On the contrary, studies into the use of BEV in the treatment of recurrent glioblastoma have reported promising results $(3,12)$. In our study, the mean score of MMSE increased more in the BEV + TMZ group than in the TMZ group. There exists some evidence that bevacizumab prolongs progression-free survival in HGGs $(13,14)$. However, the impact of this agent on quality of life yet remains to be clarified, especially with the combined use of temozolomide. In our study, with the combination therapy of bevacizumab and temozolomide, $81.6 \%$ of relapsed tumors achieved OR.

It has been reported that $\mathrm{BEV}$ could alleviate severe, even refractory cerebral edema, improve neural functions and restore functional independence (15-17). Mechanistically, $\mathrm{BEV}$ can remodel the chaotic structure of tumor blood vessels, reduce vascular permeability and interstitial fluid pressure, and reduce glucocorticoid dependence. Cognitive impairment could result from tumor-related factors (such as tumor infiltration, tumor-related edema) and treatmentrelated adverse effects. Clinically, it has been extensively observed that with the control of tumor-related edema, the cognitive state and quality of life quickly improved

Table 2 Scores of domains of QLQ-C30 in patients treated with BEV and TMZ for recurrent glioma

\begin{tabular}{lcccc}
\hline \multirow{2}{*}{ Domains } & \multicolumn{2}{c}{ BEV plus TMZ (mean \pm SD) } & Change of score & P value \\
\cline { 2 - 4 } & Baseline & Second cycle of BEV & +26.8 & 0.001 \\
PF & $34.9 \pm 12.0$ & $61.7 \pm 12.5$ & +3.8 & 0.062 \\
RF & $41.1 \pm 16.7$ & $44.9 \pm 14.2$ & +22.0 & 0.001 \\
EF & $41.4 \pm 9.2$ & $63.4 \pm 6.9$ & +28.6 & 0.001 \\
CF & $35.1 \pm 13.1$ & $63.7 \pm 12.0$ & +3.9 & 0.062 \\
SF & $44.0 \pm 15.2$ & $47.9 \pm 12.3$ & +29.2 & 0.001 \\
GHS & $39.8 \pm 6.9$ & $69.0 \pm 12.2$ & -11.5 & 0.001 \\
FA & $59.9 \pm 12.6$ & $48.4 \pm 9.2$ & -21.7 & 0.001 \\
NV & $53.8 \pm 15.1$ & $32.1 \pm 11.5$ & -19.6 & 0.001 \\
PA & $46.4 \pm 23.3$ & $26.8 \pm 10.5$ & & \\
\hline
\end{tabular}

PF, physical function; RF, role function; EF, emotional function; CF, cognitive function; SF, social function; GHS, global health status; FA, fatigue; NV, nausea/vomiting; PA, pain. 
regardless of the extent of tumor regression. In the BEV + TMZ group, the MMSE significantly increased after two cycles of BEV compared to the baseline state.

Meanwhile, cognitive function greatly improved in patients who obtained ERI higher than 0.5 , and in these patients, the MMSE increased from 19.9 to 25.0. On the contrary, there was no significant improvement in the cognitive function in patients with lower ERI. Similarly, in patients with higher ERI, the QLQ-C30 scores (PF, EF, and $\mathrm{CF}$ ) were increased due to the remission of brain edema caused by BEV use. Taken together, the results suggest that the control of edema measured by ERI is highly correlated to the improvement in cognitive state and quality of life.

Considering the potential side effects of bevacizumab has encouraged investigators to develop approaches to discern those patients likely to respond to BEV therapy from those unlikely. The most commonly used biomarkers are MRIbased imaging parameters, such as pretreatment apparent diffusion coefficient (ADC), dynamic-susceptibility contrast (DSC) with assistance from artificial intelligence as well as radiomics $(18,19)$. Obviously, the pretreatment discrimination or stratification may deprive some favorable patients of the opportunity of BEV therapy. Therefore, early response evaluation seems more reasonable. The analysis of MRI imaging data in the CABARET trial clearly showed that early MRI (after every treatment cycle) had prognostic value for overall survival (20). Likewise, in this study, we developed a surrogate imaging biomarker, ERI, to quantitatively measure the anti-edema effect of BEV as an indirect evaluation of the response of the patient to BEV therapy. Other intracranial edema quantitative analysis methodologies have been reported, such as differential quantitative T2 (DQT2) relaxometry mapping. The region of brain edema in T2WI was consistent with that of the DQT2 mapping sequence in our study. However, its robustness is limited by the variability in $\mathrm{T} 2$ measurements within the regions of interest, especially if only two echoes are collected (21).

Furthermore, it was time-consuming to scan more than five echoes on DQT2 mapping. On the other hand, the treatment planning system in the radiation oncologist workstation has a unique advantage in accurately contouring the three-dimensional structure of specific organs and lesions, including edema areas. Subsequently, the volume of brain edema can be directly and accurately quantified. Our results showed agreement with the CABARET trial, indicating that ERI after 2 cycles of BEV therapy had the predictive value for cognitive benefit from the therapy.
Those with a minor ERI (poor responder) were unlikely to gain benefit from extended cycles, and those with evident ERI (good responder) could continue the therapy to four cycles of BEV.

Our group found that patients with a higher ERI obtained remarkable MMSE and QLQ-C30 scores. With two additional cycles (4 cycles in total) of BEV therapy, cognitive state and quality of life scores tended to remain stable, and no further alleviation was observed in terms of cerebral edema, which raised concerns about the optimal administration of BEV therapy. There is controversy regarding the neurocognitive impact of $\mathrm{BEV}$ therapy, which may be related to the treatment dosage, treatment cycle, and extent of preexisting neurological injury (22). Many studies have suggested that bevacizumab plus temozolomide as a salvage therapy to recurrent glioma could reduce the edema of neural tissues and improve the health-related quality of life $(23,24)$. However, The RTOG 0825 study revealed that patients treated with bevacizumab suffered increased symptom burden and neurocognitive dysfunction over time (11). Notably, in this trial, more than half of patients $(52.2 \%)$ in the experimental group received at least 6 cycles of BEV therapy (up to 12 cycles), in comparison to 4 cycles of BEV in our study and the maximum 6 cycles in Liu et al.'s study (23). In addition, although bevacizumab has been widely used as a part of a combination chemotherapy regimen in patients with extracranial tumors, such as colorectal cancer and lung adenocarcinoma, changes in cognitive function have rarely been reported in the literature, especially in those patients with long-term survival.

After the diagnosis of recurrence, eighteen patients received re-irradiation. Disappointingly, previous treatment was completed with minimal shrinkage of the tumor mass and edema. Therefore, the combination treatment of $\mathrm{BEV}$ and $\mathrm{TMZ}$ was implemented as a subsequent therapy in 10 patients in the $\mathrm{BEV}+\mathrm{TMZ}$ group. One treatment cycle later, evident tumor regression and edema reduction were observed. This outcome was significantly promising compared to that of patients who did not undergo re-irradiation, indicating the synergistic effect between radiotherapy and a BEV/TMZ regimen. The increased permeability of the blood-brain barrier from irradiation may be favorable for drug penetration into tumors (25). However, increased permeability could worsen the edema, an unavoidable adverse effect in intracranial radiotherapy, which justifies the addition of BEV to counteract the edema.

Certainly, this study has limitations due to its 
retrospective character. The small sample number and short follow-up period may cause interpretation bias. Hence, larger sample size and longer follow-up time are required in the future study. Although this study suggests that an improvement in short-term cognitive function is related to alleviating cerebral edema, other related factors are still unclear and worth exploring. It also remains to be determined whether serious adverse effects would occur after long-term treatment with BEV, whether drug perfusion would increase with radiotherapy, and whether the combination of $\mathrm{BEV}$ and $\mathrm{TMZ}$ would inhibit vasculogenic mimicry (VM) in the microenvironment. All of the above questions remain to be further studied.

\section{Conclusions}

This study demonstrated that short-term treatment with bevacizumab (less than 4 cycles of $\mathrm{BEV}$ ) plus temozolomide in patients with recurrent high-grade gliomas was favorable to the improvement of cognitive function. Additionally, those patients with rapid improvement in cognitive function benefited more from the addition of bevacizumab and maintained longer functional improvements. However, those with poor response to $\mathrm{BEV}$ with minor cognitive improvement and small ERI value after the second cycle of BEV were highly unlikely to gain clinical benefit with extended cycles. From the perspective of imaging assessment, patients with a higher ERI benefited more from bevacizumab than patients with a lower ERI. Due to the increased risk of vascular mimicry and induced drug resistance with long-term bevacizumab treatment, timely imaging assessment and individualized treatment cycle of BEV should be recommended. Certainly, these results still need to be further validated in well-designed, randomized, and prospective clinical trials.

\section{Acknowledgments}

Funding: This research was partly supported by Grant No. 81472794 from the National Natural Science Foundation of China, Grant 2018BR23, 20184Y0099 from Shanghai Municipal Commission of Health, and Grant 17411963600 , 18441904400 from Shanghai Municipal Commission of Science and Technology.

\section{Footnote}

Conflicts of Interest: All authors have completed the
ICMJE uniform disclosure form (available at https:// dx.doi.org/10.21037/qims-20-1084). All authors reported that this research was partly supported by Grant No. 81472794 from the National Natural Science Foundation of China, Grant 2018BR23, 20184Y0099 from Shanghai Municipal Commission of Health and Grant 17411963600, 18441904400 from Shanghai Municipal Commission of Science and Technology.

Ethical Statement: The authors are accountable for all aspects of the work in ensuring that questions related to the accuracy or integrity of any part of the work are appropriately investigated and resolved. The study was conducted in accordance with the Declaration of Helsinki (as revised in 2013). The studies involving human participants were reviewed and approved by the Ethics Committee of Clinical Research of Huadong Hospital. Written informed consent from the patients/participants was not required to participate in this study in accordance with the national legislation and the institutional requirements.

Open Access Statement: This is an Open Access article distributed in accordance with the Creative Commons Attribution-NonCommercial-NoDerivs 4.0 International License (CC BY-NC-ND 4.0), which permits the noncommercial replication and distribution of the article with the strict proviso that no changes or edits are made and the original work is properly cited (including links to both the formal publication through the relevant DOI and the license). See: https://creativecommons.org/licenses/by-nc-nd/4.0/.

\section{References}

1. Ferris SP, Hofmann JW, Solomon DA, Perry A. Characterization of gliomas: from morphology to molecules. Virchows Arch 2017;471:257-69.

2. Gusyatiner O, Hegi ME. Glioma epigenetics: From subclassification to novel treatment options. Semin Cancer Biol 2018;51:50-8.

3. Friedman HS, Prados MD, Wen PY, Mikkelsen T, Schiff D, Abrey LE, Yung WK, Paleologos N, Nicholas MK, Jensen R, Vredenburgh J, Huang J, Zheng M, Cloughesy T. Bevacizumab alone and in combination with irinotecan in recurrent glioblastoma. J Clin Oncol 2009;27:4733-40.

4. Lee I, Adimadhyam S, Nutescu EA, Zhou J, Asfaw AA, Sweiss KI, Patel PR, Calip GS. Bevacizumab Use and the Risk of Arterial and Venous Thromboembolism in Patients with High-Grade Gliomas: A Nested Case-Control Study. 
Pharmacotherapy 2019;39:921-8.

5. Ciesielska N, Sokołowski R, Mazur E, Podhorecka M, Polak-Szabela A, Kędziora-Kornatowska K. Is the Montreal Cognitive Assessment (MoCA) test better suited than the Mini-Mental State Examination (MMSE) in mild cognitive impairment (MCI) detection among people aged over 60? Meta-analysis. Psychiatr Pol 2016;50:1039-52.

6. Wang Q, Xiao F, Qi F, Song X, Yu Y. Risk Factors for Cognitive Impairment in High-Grade Glioma Patients Treated with Postoperative Radiochemotherapy. Cancer Res Treat 2020;52:586-93.

7. Imber BS, Lin AL, Zhang Z, Keshavamurthy KN, Deipolyi AR, Beal K, Cohen MA, Tabar V, DeAngelis LM, Geer EB, Yang TJ, Young RJ. Comparison of Radiographic Approaches to Assess Treatment Response in Pituitary Adenomas: Is RECIST or RANO Good Enough? J Endocr Soc 2019;3:1693-706.

8. Li L, Mo FK, Chan SL, Hui EP, Tang NS, Koh J, Leung LK, Poon AN, Hui J, Chu CM, Lee KF, Ma BB, Lai PB, Chan AT, Yu SC, Yeo W. Prognostic values of EORTC QLQ-C30 and QLQ-HCC18 index-scores in patients with hepatocellular carcinoma - clinical application of health-related quality-of-life data. BMC Cancer 2017;17:8.

9. Tewari KS, Sill MW, Penson RT, Huang H, Ramondetta LM, Landrum LM, et al. Bevacizumab for advanced cervical cancer: final overall survival and adverse event analysis of a randomised, controlled, open-label, phase 3 trial (Gynecologic Oncology Group 240). Lancet 2017;390:1654-63.

10. Chinot OL, Wick W, Mason W, Henriksson R, Saran F, Nishikawa R, Carpentier AF, Hoang-Xuan K, Kavan P, Cernea D, Brandes AA, Hilton M, Abrey L, Cloughesy T. Bevacizumab plus radiotherapy-temozolomide for newly diagnosed glioblastoma. N Engl J Med 2014;370:709-22.

11. Gilbert MR, Dignam JJ, Armstrong TS, Wefel JS, Blumenthal DT, Vogelbaum MA, et al. A randomized trial of bevacizumab for newly diagnosed glioblastoma. N Engl J Med 2014;370:699-708.

12. Wenger KJ, Wagner M, You SJ, Franz K, Harter PN, Burger MC, Voss M, Ronellenfitsch MW, Fokas E, Steinbach JP, Bähr O. Bevacizumab as a last-line treatment for glioblastoma following failure of radiotherapy, temozolomide and lomustine. Oncol Lett 2017;14:1141-6.

13. Ameratunga $M$, Pavlakis $\mathrm{N}$, Wheeler $\mathrm{H}$, Grant R, Simes J, Khasraw M. Anti-angiogenic therapy for high-grade glioma. Cochrane Database Syst Rev 2018;11:CD008218.

14. Khasraw M, Simeonovic M, Grommes C. Bevacizumab for the treatment of high-grade glioma. Expert Opin Biol
Ther 2012;12:1101-11.

15. Berghoff AS, Sax C, Klein M, Furtner J, Dieckmann K, Gatterbauer B, Widhalm G, Rudas M, Zielinski CC, Bartsch R, Preusser M. Alleviation of brain edema and restoration of functional independence by bevacizumab in brain-metastatic breast cancer: a case report. Breast Care (Basel) 2014;9:134-6.

16. Takano S, Kimu H, Tsuda K, Osuka S, Nakai K, Yamamoto T, Ishikawa E, Akutsu H, Matsuda M, Matsumura A. Decrease in the apparent diffusion coefficient in peritumoral edema for the assessment of recurrent glioblastoma treated by bevacizumab. Acta Neurochir Suppl 2013;118:185-9.

17. Shen Q, Zhou L, Yao XL, Fan FF, Miao ZZ, Zhao K. Clinical observation of bevacizumab combined with temozolomide in the treatment of glioblastoma patients with intractable brain edema after radiotherapy and chemotherapy. Chin J Cancer Prev Treat 2019:26:1115-23.

18. Grossmann P, Narayan V, Chang K, Rahman R, Abrey L, Reardon DA, Schwartz LH, Wen PY, Alexander BM, Huang R, Aerts HJWL. Quantitative imaging biomarkers for risk stratification of patients with recurrent glioblastoma treated with bevacizumab. Neuro Oncol 2017;19:1688-97.

19. Petrova L, Korfiatis P, Petr O, LaChance DH, Parney I, Buckner JC, Erickson BJ. Cerebral blood volume and apparent diffusion coefficient - Valuable predictors of non-response to bevacizumab treatment in patients with recurrent glioblastoma. J Neurol Sci 2019;405:116433.

20. Field KM, Phal PM, Fitt G, Goh C, Nowak AK, Rosenthal MA, Simes J, Barnes EH, Sawkins K, Cher LM, Hovey EJ, Wheeler H. The role of early magnetic resonance imaging in predicting survival on bevacizumab for recurrent glioblastoma: Results from a prospective clinical trial (CABARET). Cancer 2017;123:3576-82.

21. Ellingson BM, Cloughesy TF, Lai A, Nghiemphu PL, Lalezari S, Zaw T, Motevalibashinaeini K, Mischel PS, Pope WB. Quantification of edema reduction using differential quantitative T2 (DQT2) relaxometry mapping in recurrent glioblastoma treated with bevacizumab. J Neurooncol 2012;106:111-9.

22. Zhang N, Chen J, Ferraro GB, Wu L, Datta M, Jain RK, Plotkin SR, Stemmer-Rachamimov A, Xu L. Anti-VEGF treatment improves neurological function in tumors of the nervous system. Exp Neurol 2018;299:326-33.

23. Liu Y, Feng F, Ji P, Liu B, Ge S, Yang C, Lou M, Liu J, Li B, Gao G, Qu Y, Wang L. Improvement of health related quality of life in patients with recurrent glioma treated 
with bevacizumab plus daily temozolomide as the salvage therapy. Clin Neurol Neurosurg 2018;169:64-70.

24. Wefel JS, Cloughesy T, Zazzali JL, Zheng M, Prados M, Wen PY, Mikkelsen T, Schiff D, Abrey LE, Yung WK, Paleologos N, Nicholas MK, Jensen R, Vredenburgh J, Das A, Friedman HS. Neurocognitive function in patients with recurrent glioblastoma treated with bevacizumab.

Cite this article as: Wang X, Chen D, Qiu J, Li S, Zheng X. The relationship between the degree of brain edema regression and changes in cognitive function in patients with recurrent glioma treated with bevacizumab and temozolomide. Quant Imaging Med Surg 2021;11(11):4556-4568. doi: 10.21037/qims-201084
Neuro Oncol 2011;13:660-8.

25. Zhang M, Jin HG, Su QX, Bu MW, Li YP, Liu GG. Changes of blood-brain barrier permeability in patients with malignant glioma after treated with VMAT radiotherapy. Journal of Jilin University (Medicine edition) 2012;38:784-7. 